\title{
KIM 3: AN ULTRA-FAINT STAR CLUSTER IN THE CONSTELLATION OF CENTAURUS
}

\author{
Dongwon Kim, Helmut Jerjen, Dougal Mackey, Gary S. Da Costa, and Antonino P. Milone \\ Research School of Astronomy and Astrophysics, Australian National University, Canberra, ACT 2611, Australia; dongwon.kim@anu.edu.au \\ Received 2015 December 10; accepted 2016 February 12; published 2016 March 29
}

\begin{abstract}
We report the discovery of an ultra-faint star cluster in the constellation of Centaurus. This new stellar system, Kim 3, features a half-light radius of $r_{h}=2.29_{-0.52}^{+1.28} \mathrm{pc}$ and a total luminosity of $M_{V}=+0.7 \pm 0.3$. Approximately 26 stars are identified as candidate member stars down to four magnitudes below the main-sequence turn-off, which makes Kim 3 the least luminous star cluster known to date. The compact physical size and extreme low luminosity place it close to faint star clusters in the size-luminosity plane. The stellar population of Kim 3 appears to be relatively young $\left(9.5_{-1.7}^{+3.0} \mathrm{Gyr}\right)$ and metal-poor $\left([\mathrm{Fe} / \mathrm{H}]=-1.6_{-0.30}^{+0.45}\right)$ at a heliocentric distance of $15.14_{-0.28}^{+1.00}$ kpc. The cluster lacks a well-defined center, and a small but prominent group of stars consistent with the Kim 3 isochrone is present approximately $9.7 \mathrm{pc}$ in projection south of the cluster center. Both are signs of the cluster being in the final stage of tidal disruption.
\end{abstract}

Key words: Galaxy: halo - globular clusters: general - globular clusters: individual (Kim 3)

\section{INTRODUCTION}

The Sloan Digital Sky Survey (SDSS; York et al. 2000) has unveiled a significant number of ultra-faint dwarf galaxies in the Milky Way halo (e.g., Willman et al. 2005; Belokurov et al. 2006; Zucker et al. 2006; Irwin et al. 2007; Walsh et al. 2007; Kim et al. 2015a). However, only a small number of new star clusters were found (Koposov et al. 2007; Belokurov et al. 2010; Balbinot et al. 2013; Kim \& Jerjen 2015a), with heliocentric distances of $17-50 \mathrm{kpc}$. These star clusters share both small physical sizes and low luminosities, properties considered to be consequences of stellar mass loss owing to internal (e.g., dynamic relaxation) and/or external (e.g., tidal stripping, tidal shocking) dynamical evolution processes (Gnedin \& Ostriker 1997; Rosenberg et al. 1998). This picture of the low-luminosity star clusters being strongly dynamically evolved is supported by growing observational evidence such as the presence of extra-tidal stars, flat luminosity functions (LFs), and substantial mass segregation (Carraro et al. 2007; Carraro 2009; Niederste-Ostholt et al. 2010; Fadely et al. 2011; Kim \& Jerjen 2015a; Kim et al. 2015b).

Since the success of SDSS, other blind imaging surveys have continued searching for new stellar systems in the Milky Way halo; the Dark Energy Survey (DES; The Dark Energy Survey Collaboration 2005), the Pan-STARRS $3 \pi$ survey (K. Chambers et al. 2016, in preparation), the VST ATLAS survey (Shanks et al. 2015), the Stromlo Milky Way Satellite (SMS) survey (Jerjen 2010), and the Survey of the Magellanic Stellar History (D. Nidever et al. 2016, in preparation). These efforts have uncovered more than 20 new satellite candidates up to the present time (Belokurov et al. 2014; Laevens et al. 2014, 2015a, 2015b; Bechtol et al. 2015; Drlica-Wagner et al. 2015; Kim \& Jerjen 2015b; Kim et al. 2015b; Koposov et al. 2015a; Luque et al. 2016; Martin et al. 2015; Torrealba et al. 2016). Spectroscopic follow-up has revealed the kinematic and chemical characteristics of some of these systems, clarifying their nature (e.g., Kirby et al. 2015a; Koposov et al. 2015b; Martin et al. 2015, 2016b; Simon et al. 2015; Walker et al. 2015 , 2016). These ultra-faint stellar systems are rapidly filling the gap between star clusters and dwarf galaxies in the size-luminosity plane, rendering this diagnostic tool less effective (e.g., see discussions in Belokurov et al. 2014; Laevens et al. 2014). Hence, deeper imaging and spectroscopic follow-up are becoming imperative to determine the true nature of the systems and possibly identify star clusters among the new candidates (e.g., Kirby et al. 2015b; Weisz et al. 2015)

In this paper, we announce the discovery of a new ultra-faint star cluster, which we designate as $\operatorname{Kim} 3$, found in the constellation of Centaurus. This concentration of stars was detected as part of our ongoing imaging survey with the Dark Energy Camera (DECam) on the $4 \mathrm{~m}$ Blanco telescope at Cerro Tololo in Chile. Section 2 describes the observations and data reduction process, including photometry and star/galaxy separation that led to the discovery of Kim 3 . We also discuss the photometric calibration and completeness tests. Section 3 contains our analysis of the color-magnitude diagram (CMD) and describes how we derived the properties of the new star cluster such as age, metallicity, distance, luminosity and structure. We discuss the results and draw our conclusions in Section 4.

\section{OBSERVATION, DATA REDUCTION, AND DISCOVERY}

As part of the SMS Survey project, we have observed in non-targeted mode 500 square degrees of sky in the Centaurus region (see Figure 1) using the DECam (Flaugher et al. 2015) of the $4 \mathrm{~m}$ Blanco Telescope located at Cerro Tololo Inter-American Observatory. The imager consists of 62 $2 \mathrm{k} \times 4 \mathrm{k}$ CCD chips with a pixel scale of 0 !" 27, which delivers an $\sim 3$ square degree field of view. We obtained images in the $g$ and $r$ bands over two observing runs in 2014 July and 2015 June as part of observing proposals 2014A-0624 and 2015A0616 (both PI: H. Jerjen). More details on the former observing run can be found in our previous work (Kim et al. 2015b). In the case of the latter session, we set the exposure times to between 100 and $210 \mathrm{~s}$ depending on the fraction of moon illumination and the angular distance of the target field from the moon. To fill the inter-chip gaps, we dithered in a diagonal direction by half of a single chip in both $x$ and $y$ for each field, providing two exposures per field per filter. The images were reduced using the DECam community pipeline (Valdes 


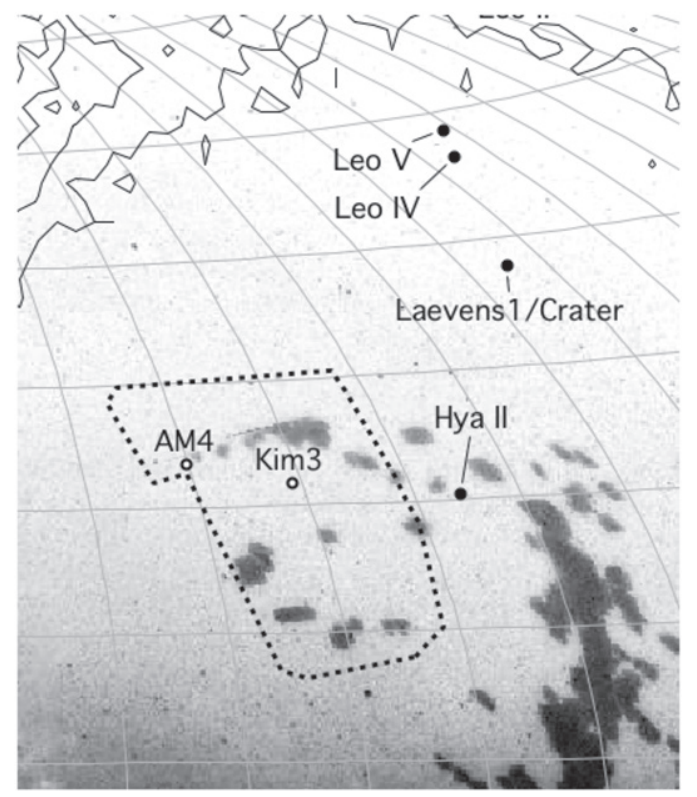

Figure 1. Section of the sky (dashed line) showing the footprint of the $\sim 500$ square degree area searched for new ultra-faint Milky Way stellar systems as part of the Stromlo Milky Way Satellite Survey. The Galactic longitude and latitude lines are spaced by $10^{\circ}$. Known satellite galaxies and star clusters in the area are labelled. Black contour lines indicate particle densities for the simulated Sagittarius stream from Law \& Majewski (2010). The background image, showing the end of a leading arm of the Magellanic Stream, is by Nidever et al. (2010), NRAO/AUI/NSF and Meilinger, Leiden-ArgentineBonn Survey, Parkes Observatory, Westerbork Observatory, Arecibo Observatory (see http://www.nrao.edu/pr/2010/magstream/).

et al. 2014). This process includes bias subtraction, dark and flat-field corrections, and the application of a WCS solution to each image.

We carried out point-spread function (PSF) photometry over the pre-processed single exposure images to produce photometric catalogs using SExtractor/PSFEx (Bertin \& Arnouts 1996; Bertin 2011) on a local 16 node/128 core computer cluster. For the star/galaxy separation we made use of the SPREAD_MODEL parameter provided by SExtractor, where the threshold was set to $\mid$ SPREAD_MODEL $\mid<0.003+$ SPREADERR_MODEL as described in Koposov et al. (2015a). This process was applied to the photometric band that exhibited the better defined PSF over the entire field. The $g$ - and $r$-band catalogs were crossmatched using STILTS (Taylor 2005) with a $1^{\prime \prime}$ tolerance. The instrumental magnitudes were then calibrated with respect to the APASS $^{1}$ DR 8 star catalog via bootstrap sampling with 500 iterations and $3 \sigma$ clipping. The number of matched stars in a field ranged between 100 and 1600. Finally, each calibrated object was corrected for Galactic extinction based on the reddening map by Schlegel et al. (1998) and the correction coefficients from Schlafly \& Finkbeiner (2011).

We ran our overdensity detection algorithm, which is based on the method of Walsh et al. (2009), over the final point source catalog that was produced for each field by our photometry pipeline. For more details about the algorithm, see Kim \& Jerjen (2015a). Briefly, the algorithm enhances the contrast between satellite population and the Milky Way foreground stars by using photometric filters in the colormagnitude space and comparing the integrated signal-to-noise

1 https://www.aavso.org/apass

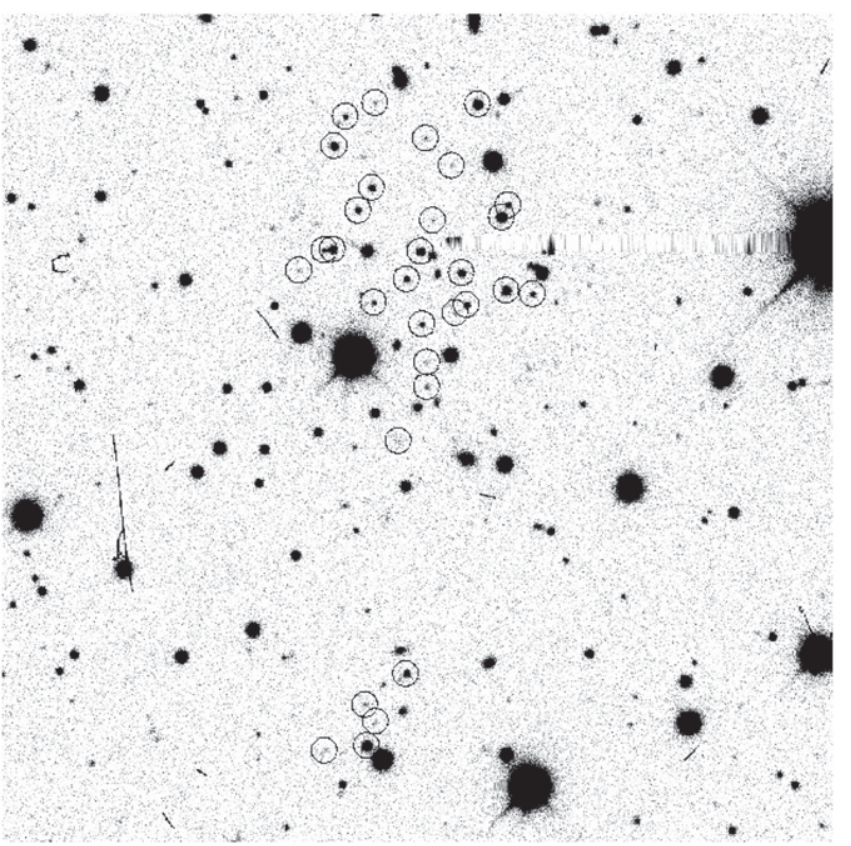

Figure 2. $4 \times 4 \operatorname{arcmin}^{2}$ DECam cutout $r$-band image of Kim 3 with $210 \mathrm{~s}$ exposure time. North is up, east is to the left. Circled are all stars fainter than $r_{0}=18.5$ that are within two half-light radii from the adopted center of Kim 3 or within a radius of 0.15 from the adopted center of the small group of stars to the south of Kim 3 (see Figure 3), and consistent with the best-fitting isochrone $(9.5 \mathrm{Gyr},[\mathrm{Fe} / \mathrm{H}]=-1.6)$ at a distance of $15.14 \mathrm{kpc}$.

ratios (S/Ns) of point-source clusters on a convolved stellar density map in the field of view of DECam. In this search, we recovered the known globular cluster AM4 and detected the new object Kim 3, the $\mathrm{S} / \mathrm{N}$ of which reached the $10 \sigma$ level over the Poisson noise measured in the surrounding point-source distribution.

We performed completeness tests for the photometry as follows. We first created an accurate PSF model image using the PSF task of DAOPHOT in the IRAF environment and then added 100 artificial stars per chip at random pixel coordinates using the ADDSTAR task in IRAF. A series of images were produced for different input magnitudes at 0.5 mag intervals. We then ran our photometry routine and measured the recovery rate, for which we also applied the same star/galaxy separation criteria for more realistic measurements. This procedure was repeated 20 times to obtain reliable statistics. The completeness function for our CMD was then finalized by multiplying the recovery rates in the $g$ and $r$ bands as the two catalogs were cross-matched to generate the CMD. The $90 \%$ and $50 \%$ levels of our photometry at the color $(g-r)=0.5$ correspond to $r=20.74$ and $r=23.21$ respectively. The $50 \%$ completeness level as a function of color and $r$ magnitude is indicated by the dotted lines in Figure 4.

Figure 2 shows an $r$-band cutout image centered on Kim 3, where the cluster is completely resolved into individual stars. We note that a very bright star to the west of the cluster caused a "blooming" effect across the image, which was automatically corrected via linear interpolation by the NOAO community pipeline. It is possible that some Kim 3 member stars are hidden behind the interpolated region. Another bright star to the south-east of the nominal cluster center could also be hiding stars associated with the cluster. 

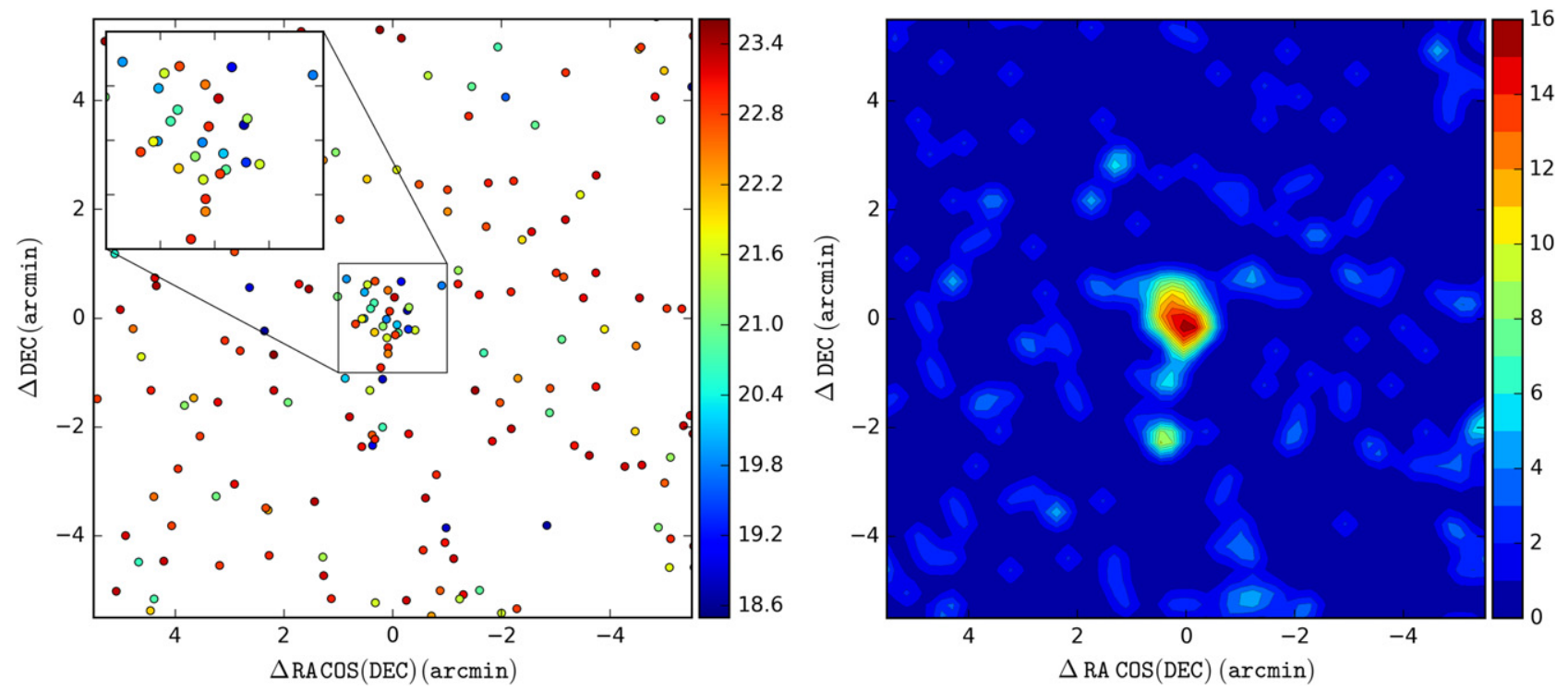

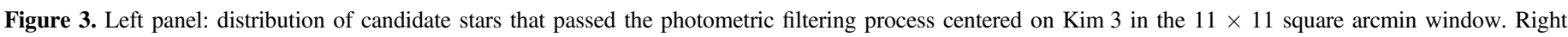

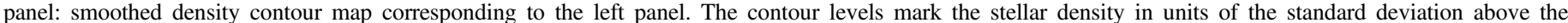
background.

The left panel of Figure 3 shows the R.A.-decl. distribution of all stars that passed the photometric filter based on the main sequence and its turn off of the best-fitting isochrone. The color of the marker represents the $r_{0}$ magnitude. The right panel shows the corresponding convolved density map where the contours are in units of the measured standard deviation. The contours reveal an asymmetric feature within high density level isophotes $(>6 \sigma)$ and a tail-like structure to the south, which could be evidence of tidal disruption. We note that a similar feature has been found in the case of the dissolving star cluster Kim 1 (see Figure 2 in Kim \& Jerjen 2015a). At the end of that structure, a small but prominent overdensity clearly stands out $\sim 2 ! 2$ below the center of $\mathrm{Kim} 3$. This feature further strengthens the impression that the tail is actually the product of the disruption process in the cluster.

\section{KIM 3 PROPERTIES}

\subsection{Color-Magnitude Diagram}

The left panel of Figure 4 shows all star-like objects from our analysis found in the vicinity of Kim 3 where blue markers represent objects in common in all four exposures, $2 \times 100 \mathrm{~s}$ in $g$ band and $2 \times 210 \mathrm{~s}$ in $r$ band. The next three panels on the right correspond to the CMD of stars within two half-light radii of Kim 3, the control CMD and the differential Hess diagram. We calculated the uncertainty weighted average magnitudes for the overlapping objects so that their photometric uncertainties are $\sim 30 \%$ smaller than those with the single measurements. The CMD of Kim 3 possesses stars over $\sim 4 \mathrm{mag}$, from the MSTO down to the $50 \%$ completeness level, that are consistent with an old $(9.5 \mathrm{Gyr})$ and metal-poor $([\mathrm{Fe} / \mathrm{H}]=-1.6)$ population at a distance modulus of $(m-M)=15.90$. Such a tight main-sequence fit has also been noticed in the CMD of Kim 1 . The CMD of the smaller overdensity nearby Kim 3 also shows a fairly consistent fit to the same isochrone within photometric uncertainties. Its true affilliation to Kim 3 can be determined once spectroscopic data become available.

\subsection{Age, Metallicity, and Distance Modulus}

We estimate the age, metallicity, and distance of Kim 3 using the maximum likelihood (ML) method described in Frayn \& Gilmore (2002), Fadely et al. (2011), and Kim \& Jerjen (2015a). For the analysis, we use all stars within an elliptical radius of 1!0 from Kim 3, the inner ellipse in the upper left panel of Figure 4. We calculate the ML values as defined by Equations 1 and 2 in Fadely et al. (2011), over a grid of Dartmouth model isochrones (Dotter et al. 2008). The grid points in the multi-dimensional parameter space cover the age range from 7.0 to $13.5 \mathrm{Gyr}$, a metallicity range of $-2.5 \leqslant[\mathrm{Fe} / \mathrm{H}] \leqslant-0.5 \mathrm{dex}$, and a distance range of $15.7<(m-M)<16.3$. Grid steps are $0.5 \mathrm{Gyr}$, $0.1 \mathrm{dex}$, and $0.05 \mathrm{mag}$, respectively.

Due to the small sample size relative to the number of free parameters, we chose to fix $[\alpha / \mathrm{Fe}]$ in the fit to ensure adequate convergence of the ML algorithm. We tested two scenarios - the first with $[\alpha / \mathrm{Fe}]=+0.4$ to match most known Galactic globular clusters, and the second with $[\alpha / \mathrm{Fe}]=0.0$ to match the small sample of younger globular clusters seen in the MW halo (e.g., Cohen 2004; Sbordone et al. 2005; Sakari et al. 2011; Villanova et al. 2013). We found a similar age and distance modulus for each scenario, but rather different values of metallicity: age $=9.5_{-1.0}^{+1.8} \mathrm{Gyr},(m-M)=15.93_{-0.03}^{+0.11} \mathrm{mag}$, and $[\mathrm{Fe} / \mathrm{H}]=-2.0_{-0.40}^{+0.35}$ for $[\alpha / \mathrm{Fe}]=+0.4$, and $9.5_{-1.7}^{+3.0} \mathrm{Gyr}$, $(m-M)=15.90_{-0.04}^{+0.11} \mathrm{mag}$, and $[\mathrm{Fe} / \mathrm{H}]=-1.6_{-0.30}^{+0.45}$ for $[\alpha / \mathrm{Fe}]=0.0$. With the first solution, $\operatorname{Kim} 3$ would be a significant outlier in the age-metallicity relationship observed for Galactic globular clusters (see Figure 10 in Dotter et al. 2011), but with the second it would agree much more closely. Given that Kim 3 appears to have a relatively young age, we adopt the solution for $[\alpha / \mathrm{Fe}]=0.0$ as our final estimate for the rest of the paper. However, we will ultimately need spectroscopic follow-up to confirm these results.

In Figure 5, we present the matrix of likelihood values for the sample described above after interpolation and smoothing over two grid points. The best-fitting Dartmouth isochrone (red solid line in Figure 4) has an age of $9.5 \mathrm{Gyr},[\mathrm{Fe} / \mathrm{H}]=-1.6$ dex, $[\alpha / \mathrm{Fe}]=0.0$ with a heliocentric distance of $15.14 \mathrm{kpc}$ 

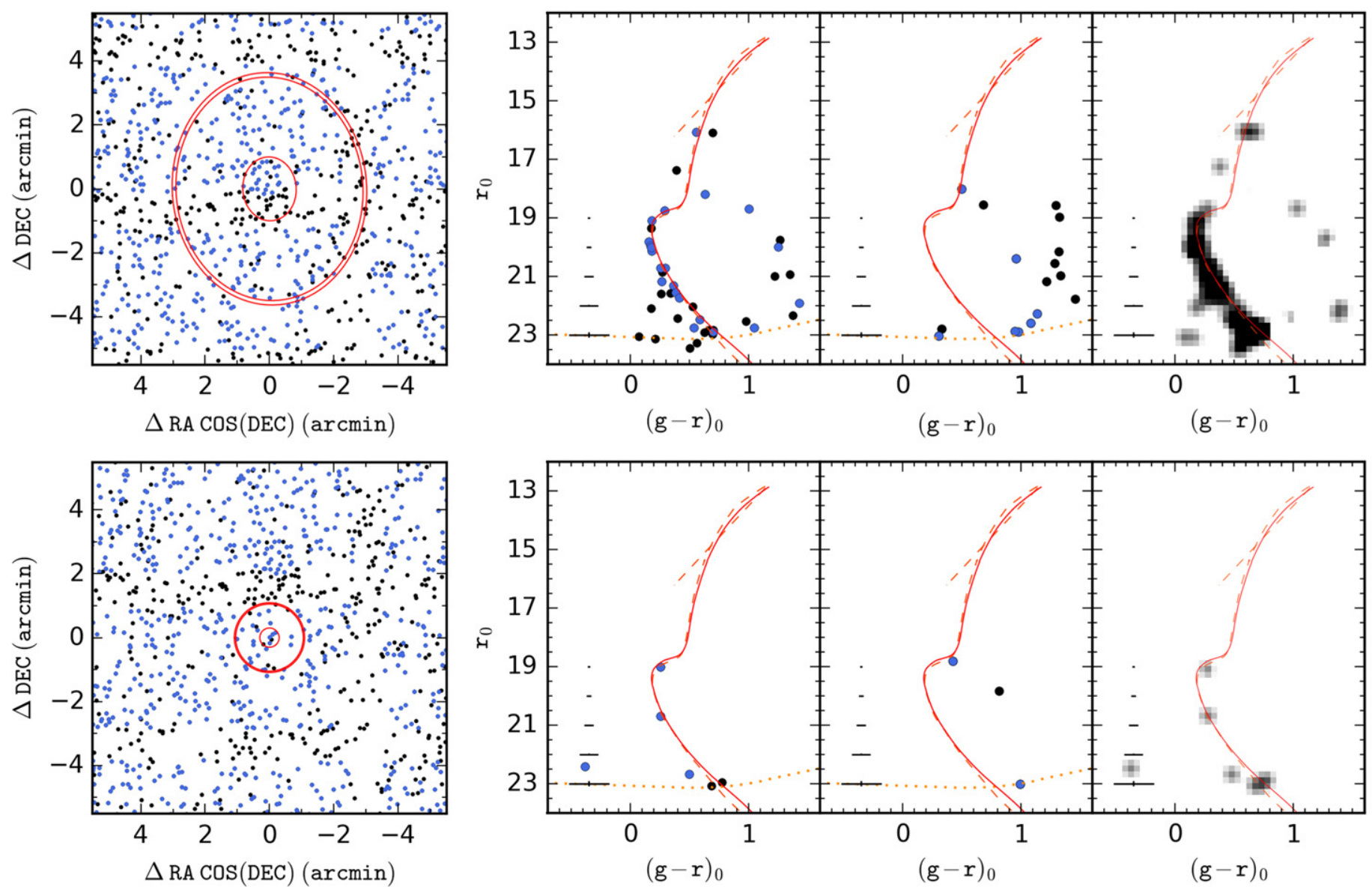

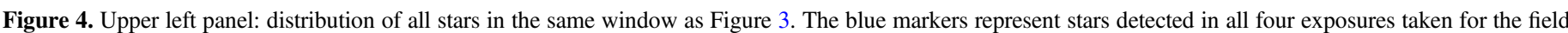

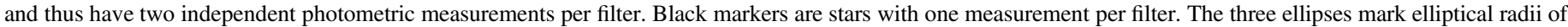

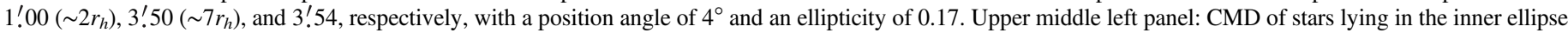

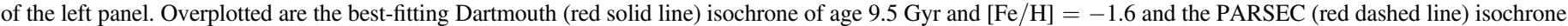

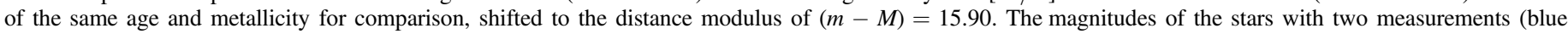

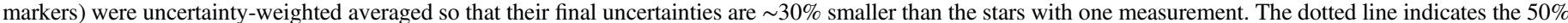

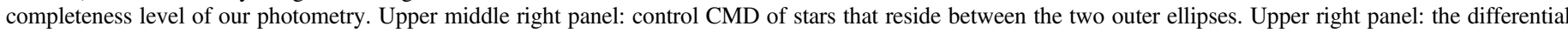

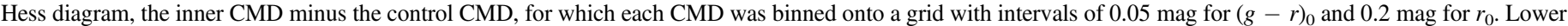

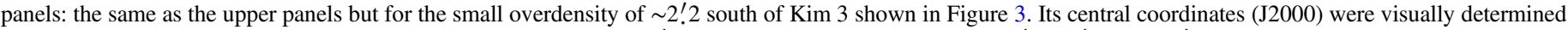
as $($ R.A., decl. $)=\left(200^{\circ} 696,-30^{\circ} .637\right)$. Its radius was measured $\sim 0 ! 15$. The three circles mark radii of $0 ! 30,1 ! 05$, and $1 ! 09$.

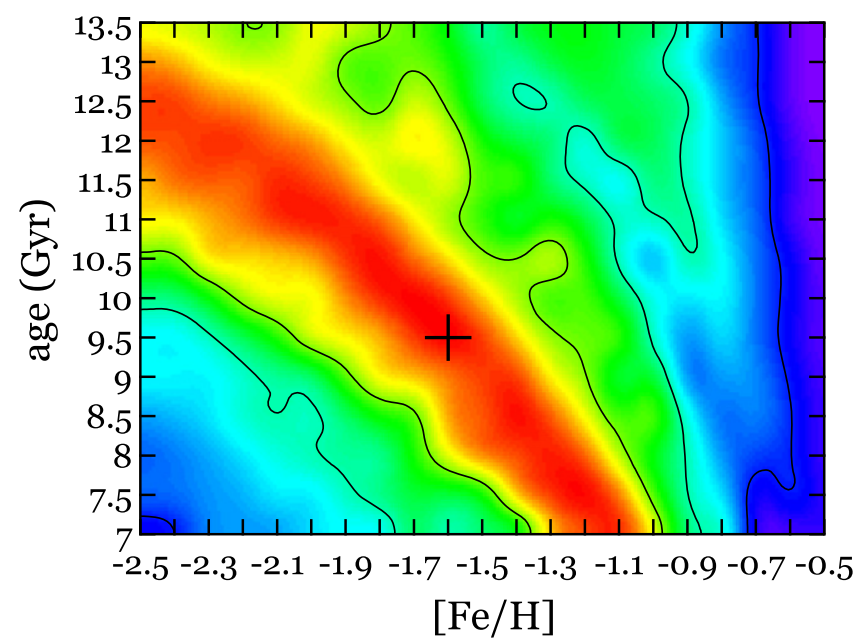

Figure 5. Smoothed maximum likelihood density map in age-metallicity space for all stars within $2 r_{h}$ around Kim 3. Contour lines show the $68 \%, 95 \%$, and $99 \%$ confidence levels. The diagonal flow of the contour lines reflects the agemetallicity degeneracy inherent to such an isochrone fitting procedure. The 1D marginalized parameters around the best fit with uncertainties are listed in Table 1.
( $m-M=15.90 \mathrm{mag})$. These estimates also yield a consistent fit for the PARSEC model isochrones (red dashed line in Figure 4). The 68\%, 95\%, and 99\% confidence contours are overplotted in Figure 5.

\subsection{Structural Parameters and Luminosity}

To determine the central coordinates and structural parameters of Kim 3, we employed the ML routine introduced in Martin et al. (2008) using the stars fainter than $r_{0}=18.5 \mathrm{mag}$ that passed the photometric filtering process. The upper panels of Figure 6 show the resulting marginal probability distribution functions (PDFs) for the structural parameters and the bottom panel shows the radial density profile with the exponential profile using the modal values from the ML analysis. Formally, Kim 3 is mildly elliptical with $\epsilon=0.17_{-0.17}^{+0.26}$ at a position angle of $\theta=4 \pm 24^{\circ}$; however, as is evident from the PDFs in Figure 5 , these quantities are not well constrained by the available data. The physical half-light radius of the cluster is calculated as $r_{h}=2.29_{-0.52}^{+1.28} \mathrm{pc}$ adopting the heliocentric distance of $15.14_{-0.28}^{+1.00} \mathrm{kpc}$ derived in Section 3.2. This shows that Kim 3 is similar in size to Segue 3 (Fadely et al. 2011). We 

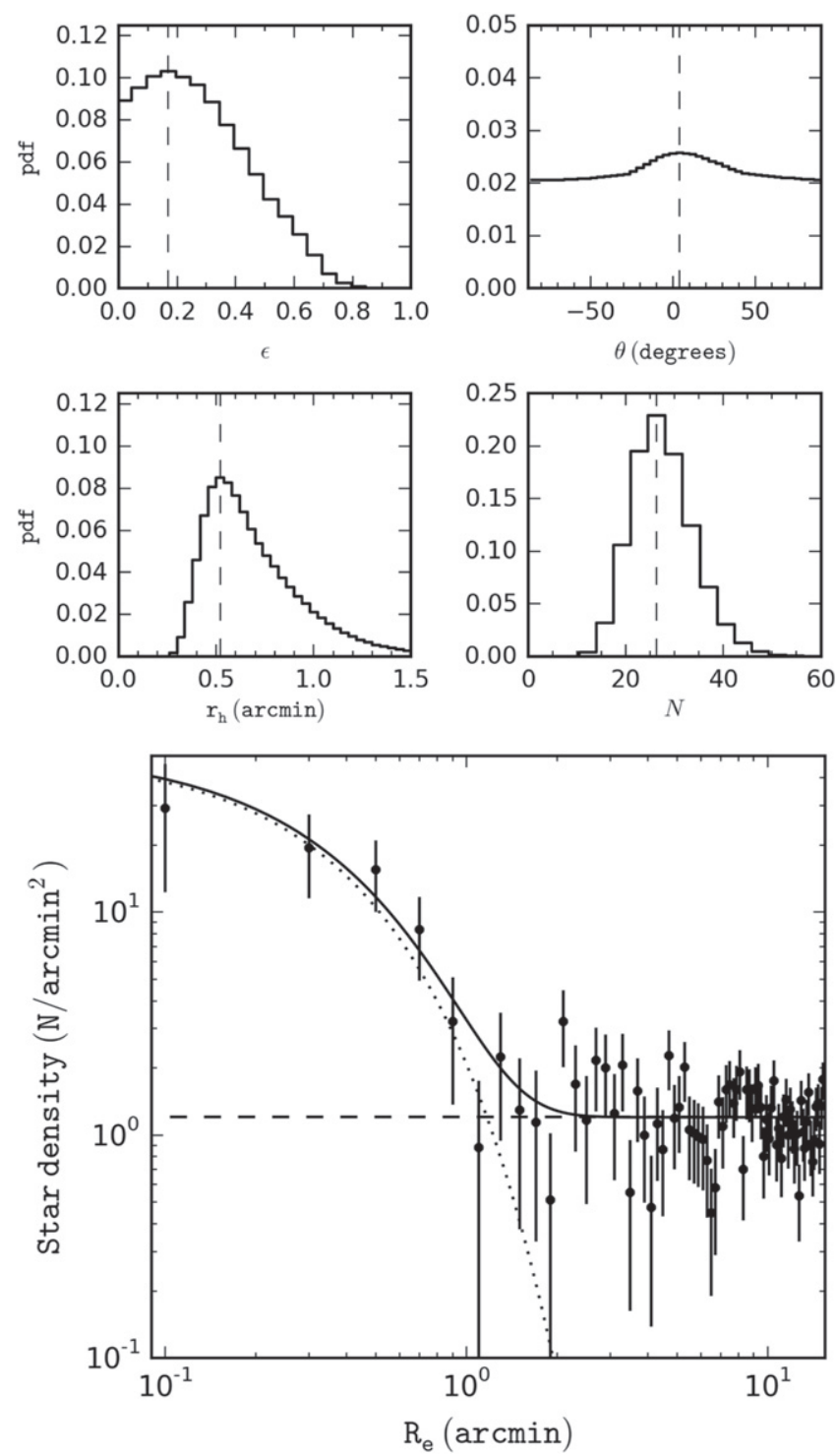

Figure 6. Upper panels: marginalized probability distribution functions for the structural parameters of $\operatorname{Kim} 3$ : the ellipticity $(\epsilon)$, the position angle $(\theta)$, halflight radius $\left(r_{h}\right)$, and the number of stars that belong to the cluster in our photometry $(N)$. The mode for each parameter is marked by a vertical dashed line. Bottom panel: radial density profile of Kim 3 based on mode values as a function of elliptical radius $R_{e}$. The dotted, dashed, and solid lines correspond to the best-fit exponential model, the foreground level and the combined fit respectively.

note that the exclusion of possible member stars obscured by the blooming effect or the bright star near the cluster (see Figure 2) could slightly affect the results. The number of stars that belong to the cluster $N$ was calculated with Equation (5) in Martin et al. (2008).

We estimated the total luminosity of Kim 3 using the star count parameter $N$ as follows. We first multiplied the normalized theoretical LF with the completeness function determined in Section 2. We then integrated the LF as a probability density function of magnitude. The ratio of the star count mode $N=26$ to the probability density gives the scale factor to transform the original LF to the observed level. Finally, we calculated the weighted integral of flux treating the scaled LF as a weight function. We obtained
Table 1

Properties of Kim 3

\begin{tabular}{lcc}
\hline \hline Parameter & Value & Unit \\
\hline$\alpha_{J 2000}$ & $132245.2 \pm 2.0$ & $\mathrm{~h} \mathrm{~m} \mathrm{~s}$ \\
$\delta_{J 2000}$ & -3036 & \\
$l$ & $03.6 \pm 2.0$ & $\mathrm{deg}$ \\
$b$ & 310.860 & $\mathrm{deg}$ \\
$(m-M)$ & 31.788 & $\mathrm{mag}$ \\
{$[\mathrm{Fe} / \mathrm{H}]$} & $15.90_{-0.04}^{+0.11}$ & $\mathrm{dex}$ \\
$d_{\odot}$ & $-1.6_{-0.30}^{+0.45}$ & $\mathrm{kpc}$ \\
$d_{\text {gal }}$ & $15.14_{-0.28}^{+1.00}$ & $\mathrm{kpc}$ \\
$r_{h}$ & $12.58_{-0.25}^{+0.85}$ & \\
$r_{h}$ & $0.52_{-0.11}^{+0.24}$ & $\mathrm{pc}$ \\
$\epsilon$ & $2.29_{-0.52}^{+1.28}$ & $\cdots$ \\
$\theta$ & $0.17_{-0.17}^{+0.26}$ & $\mathrm{deg}$ \\
$M_{V}$ & $4 \pm 24$ & $\mathrm{mag}$ \\
$E(B-V)^{\mathrm{a}}$ & $+0.7 \pm 0.3$ & $\mathrm{mag}$ \\
\hline
\end{tabular}

Note.

${ }^{\text {a }}$ From Schlafly \& Finkbeiner (2011).

$M_{r}=+0.51+_{-0.29}^{0.27}$ using the Dartmouth LF of $9.5 \mathrm{Gyr}$ and $[\mathrm{Fe} / \mathrm{H}]=-1.6$ with the mass function by Chabrier (2001) and $M_{r}=+0.43+_{-0.30}^{0.28}$ using the PARSEC LF of the same age and metallicity with the mass function by Kroupa (2001). The quoted errors include the uncertainties in the star count parameter $N$ and the distance modulus derived in Section 3.2. For a $9.5 \mathrm{Gyr}$ and $[\mathrm{Fe} / \mathrm{H}]=-1.6$ stellar population, the Dartmouth and PARSEC models have mean colors of $V-r=0.21$ and $V-r=0.22$, respectively, which convert both the $M_{r}$ magnitudes into $M_{V}=+0.7 \pm 0.3$. We adopted $M_{V}=+0.7 \pm 0.3$ as our final estimate of the total luminosity of Kim 3. All derived parameters presented in this section are summarized in Table 1.

\section{DISCUSSION AND CONCLUSION}

We report the discovery of the ultra-faint star cluster Kim 3 in the constellation of Centaurus. It is a compact $\left(r_{h}=2.29_{-0.52}^{+1.28}\right)$ and extremely faint $\left(M_{V}=+0.7 \pm 0.3\right)$ star cluster. Although its physical size and ellipticity are comparable to Segue 3, the new cluster exhibits even more asymmetry on the contour map than Segue 3 (see Figure 5 in Fadely et al. 2011). Kim 3 is even slightly fainter than $\operatorname{Kim} 1\left(M_{V}=+0.3 \pm 0.5 ; \mathrm{Kim} \&\right.$ Jerjen 2015a), Segue $3\left(M_{V}=+0.0 \pm 0.8\right.$; Fadely et al. 2011), and Muñoz $1\left(M_{V}=-0.4 \pm 0.9\right.$ Muñoz et al. 2012) and thus sets a new record in the size-luminosity plane. The best-fitting model isochrone in the CMD indicates that the stars of Kim 3 are located at a heliocentric distance of $15.14_{-0.28}^{+1.00} \mathrm{kpc}$, or a Galactocentric distance of $12.58_{-0.23}^{+0.85} \mathrm{kpc}$, and feature a metallicity $\left([\mathrm{Fe} / \mathrm{H}]=-1.6_{-0.30}^{+0.45}\right)$ and intermediate age $\left(9.5_{-1.7}^{+3.0} \mathrm{Gyr}\right)$. At the Galactic latitude of $31^{\circ} .788, \mathrm{Kim} 3$ is located $\sim 8 \mathrm{kpc}$ above the Galactic plane and is, therefore, unlikely to be an old open (disk) cluster.

The CMD of Kim 3 in Figure 4 appears to have a tight main sequence, which implies the absence of binary stars with large mass ratios. This is in contrast with the observations of a strong anti-correlation between the fraction of binaries and the mass of the cluster (Milone et al. 2012, 2016). Such a low binary fraction is more likely to be observed in the outer region of 

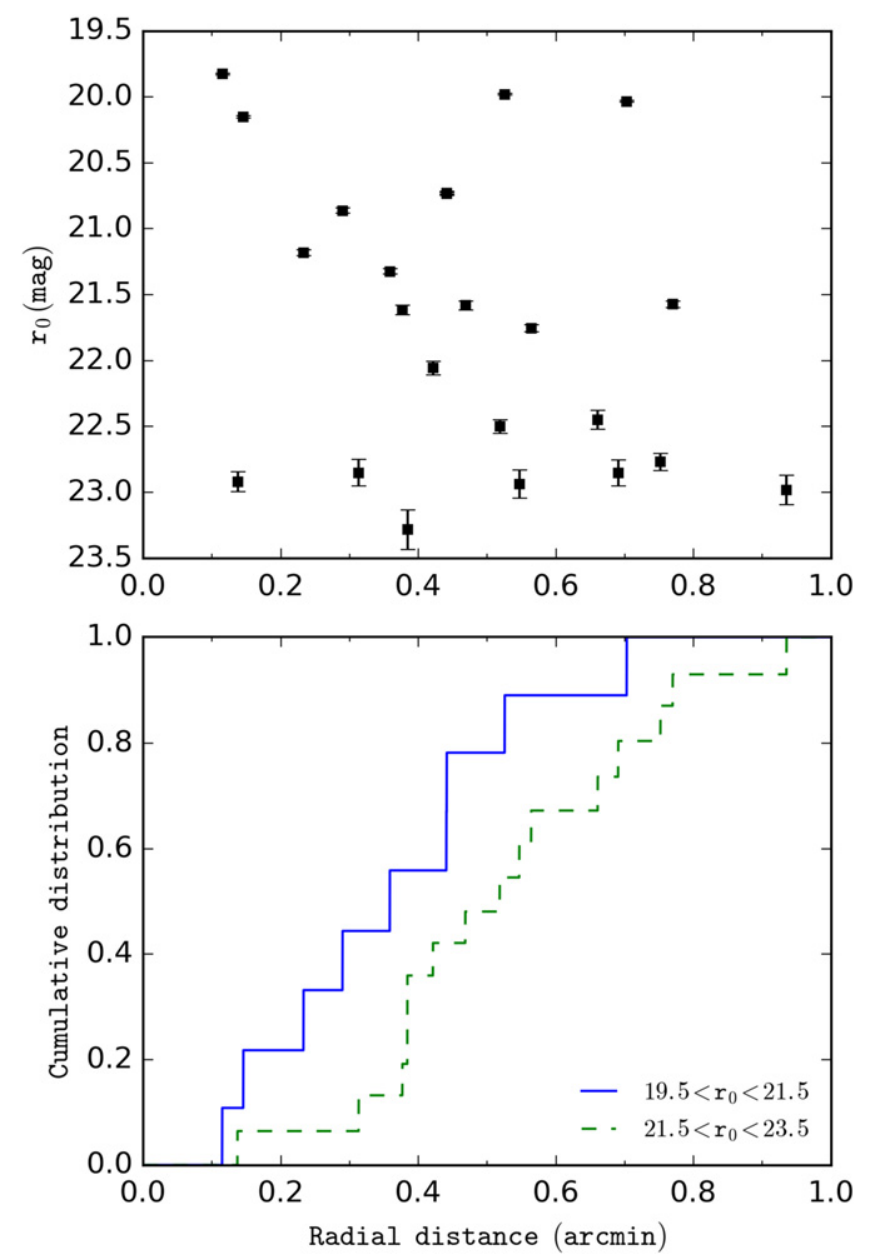

Figure 7. Upper panel: $r_{0}$ magnitudes of all Kim 3 main-sequence stars within $2 r_{h}(\sim 1 ! 0)$ as a function of radial distances from the center of the cluster. Lower panel: completeness-corrected cumulative distribution functions for two different magnitude intervals.

GCs as the binaries preferentially occupy the central region. Although the lack of a binary sequence in Figure 4 might be the consequence of low number statistics, it implies that Kim 3 possibly originated from the outskirts of a more massive GC undergoing tidal disruption in the gravitational field of the Milky Way. High precision photometry and proper-motion measurements will be able to test this hypothesis.

The half-mass relaxation time of $\operatorname{Kim} 3$ is estimated as $\sim 50 \mathrm{Myr}$ based on our measurements of the structural parameters in Section 3.3 and the Equation (5) from Spitzer \& Hart (1971). As this timescale is significantly shorter than the observed age of Kim $3\left(9.5_{-1.7}^{+3.0} \mathrm{Gyr}\right)$, it is highly likely that the cluster has been dynamically relaxed for a long time and bears evidence of mass segregation. The left panel of Figure 3 already gives an impression of mass segregation in Kim 3 in the way that the majority of bright main-sequence stars between $20.0<r_{0}<21.5$ preferentially occupy the inner region of the cluster while the fainter, less massive MS stars mainly comprise the outer part of the cluster. The top panel of Figure 7 shows the $r_{0}$ magnitudes of the 22 stars in the magnitude interval $19.5<r_{0}<23.5$ consistent with the main sequence of the best-fit isochrone in the CMD within two half-light radii $(\sim 1 ! 0)$ as a function of radial distance from the center of the cluster. The lower panel shows the corresponding cumulative distributions for two different magnitude intervals $\left(19.5<r_{0}<21.5,21.5<r_{0}<23.5\right)$, corrected for incompleteness. Although it appears that the brighter (or more massive) main-sequence stars are more common in the center of Kim 3 than in its outskirts, a Kolmogorov-Smirnov test implies that this seemingly mass-segregated state is not highly significant, yielding a formal probability of $87 \%$ that the two groups were sampled from populations with different parent distributions. This is possibly because of the relatively small sample sizes. The lack of a well defined center in the ultra-faint star cluster with an old stellar population also suggests that Kim 3 might have experienced substantial mass loss owing to tidal disruption in the gravitational field of the Milky Way (see, e.g., discussion in Kim \& Jerjen 2015a). The small overdensity 9.7 pc away from Kim 3 to the south is likely debris of the tidal disruption. We can use the centers of Kim 3 and the overdensity as reference points to determine the associated great circle. Taking it as an approximation for the orbital path of Kim 3, we find that the two globular clusters $\omega$ Centauri $\left(d_{\mathrm{gc}}=6.4 \mathrm{kpc}\right)$ and NGC5286 $\left(d_{\mathrm{gc}}=8.4 \mathrm{kpc}\right)$, which are $\sim 16^{\circ} .9$ and $\sim 21^{\circ} .2$ away from Kim 3 , are only $2^{\circ} .5$ and 0.2 away from that great circle. In this context, it is further interesting to note that these systems are among the few MW globular clusters showing internal variations in metals (Marino et al. 2015), which led to the hypothesis that they are surviving remnants of tidally disrupted dwarf galaxies. Kim 3 may have originated from a more massive stellar system that also hosted NGC5286 or Omega Centauri. Future radial velocity and proper motion measurements will help to test this idea.

The authors thank Tammy Roderick, Gyuchul Myeong, and Alfredo Zenteno for their assistance during the DECam observing run, and Aaron Dotter for his help with the Dartmouth isochrones. We also thank the referee for helpful comments and suggestions on the original manuscript. H.J. and G.D.C. acknowledge the support of the Australian Research Council through Discovery project DP150100862.

This paper makes use of data from the AAVSO Photometric All Sky Survey, whose funding has been provided by the Robert Martin Ayers Sciences Fund. This research made use of Astropy, a community-developed core Python package for Astronomy (Astropy Collaboration et al. 2013), and the Matplotlib library (Hunter 2007).

This project used data obtained with the Dark Energy Camera (DECam), which was constructed by the Dark Energy Survey (DES) collaborating institutions: Argonne National Lab, University of California Santa Cruz, University of Cambridge, Centro de Investigaciones Energeticas, Medioambientales y Tecnologicas-Madrid, University of Chicago, University College London, DES-Brazil consortium, University of Edinburgh, ETH-Zurich, Fermi National Accelerator Laboratory, University of Illinois at Urbana-Champaign, Institut de Ciencies de l'Espai, Institut de Fisica d'Altes Energies, Lawrence Berkeley National Lab, Ludwig-Maximilians Universitat, University of Michigan, National Optical Astronomy Observatory, University of Nottingham, Ohio State University, University of Pennsylvania, University of Portsmouth, SLAC National Lab, Stanford University, University of Sussex, and Texas A\&M University. Funding for DES, including DECam, has been provided by the U.S. Department of Energy, National Science Foundation, Ministry of Education and Science (Spain), Science and Technology Facilities 
Council (UK), Higher Education Funding Council (England), National Center for Supercomputing Applications, Kavli Institute for Cosmological Physics, Financiadora de Estudos e Projetos, Fundação Carlos Chagas Filho de Amparo a Pesquisa, Conselho Nacional de Desenvolvimento Cientifico e Tecnológico and the Ministrio da Ciência e Tecnologia (Brazil), the German Research Foundation-sponsored cluster of excellence "Origin and Structure of the Universe," and the DES collaborating institutions.

\section{REFERENCES}

Astropy Collaboration, Robitaille, T. P., Tollerud, E. J., et al. 2013, A\&A, 558, A33

Balbinot, E., Santiago, B. X., da Costa, L., et al. 2013, ApJ, 767, 101

Bechtol, K., Drlica-Wagner, A., Balbinot, E., et al. 2015, ApJ, 807, 50

Belokurov, V., Irwin, M. J., Koposov, S. E., et al. 2014, MNRAS, 441, 2124

Belokurov, V., Walker, M. G., Evans, N. W., et al. 2010, ApJL, 712, L103

Belokurov, V., Zucker, D. B., Evans, N. W., et al. 2006, ApJL, 647, L111

Bertin, E. 2011, adass XX, 442, 435

Bertin, E., \& Arnouts, S. 1996, A\&AS, 117, 393

Bressan, A., Marigo, P., Girardi, L., et al. 2012, MNRAS, 427, 127

Carraro, G. 2009, AJ, 137, 3809

Carraro, G., Zinn, R., \& Moni Bidin, C. 2007, A\&A, 466, 181

Chabrier, G. 2001, ApJ, 554, 1274

Cohen, J. G. 2004, AJ, 127, 1545

Dotter, A., Chaboyer, B., Jevremović, D., et al. 2008, ApJS, 178, 89

Dotter, A., Sarajedini, A., \& Anderson, J. 2011, ApJ, 738, 74

Drlica-Wagner, A., Bechtol, K., Rykoff, E. S., et al. 2015, ApJ, 813, 109

Fadely, R., Willman, B., Geha, M., et al. 2011, AJ, 142, 88

Flaugher, B., Diehl, H. T., Honscheid, K., et al. 2015, AJ, 150, 150

Frayn, C. M., \& Gilmore, G. F. 2002, MNRAS, 337, 445

Gnedin, O. Y., \& Ostriker, J. P. 1997, ApJ, 474, 223

Hunter, J. D. 2007, CSE, 9, 90

Irwin, M. J., Belokurov, V., Evans, N. W., et al. 2007, ApJL, 656, L13

Jerjen, H. 2010, AdAst, 2010, 434390

Kim, D., \& Jerjen, H. 2015a, ApJ, 799, 73

Kim, D., \& Jerjen, H. 2015b, ApJL, 808, L39

Kim, D., Jerjen, H., Mackey, D., Da Costa, G. S., \& Milone, A. P. 2015a, ApJL, 804, L44

Kim, D., Jerjen, H., Milone, A. P., Mackey, D., \& Da Costa, G. S. 2015b, ApJ, 803,63

Kirby, E. N., Cohen, J. G., Simon, J. D., \& Guhathakurta, P. 2015a, ApJL, 814, L7

Kirby, E. N., Simon, J. D., \& Cohen, J. G. 2015b, ApJ, 810, 56

Koposov, S., de Jong, J. T. A., Belokurov, V., et al. 2007, ApJ, 669, 337

Koposov, S. E., Belokurov, V., Torrealba, G., \& Evans, N. W. 2015a, ApJ, 805,130
Koposov, S. E., Casey, A. R., Belokurov, V., et al. 2015b, ApJ, 811, 62 Kroupa, P. 2001, MNRAS, 322, 231

Laevens, B. P. M., Martin, N. F., Bernard, E. J., et al. 2015a, ApJ, 813, 44

Laevens, B. P. M., Martin, N. F., Ibata, R. A., et al. 2015b, ApJL, 802, L18

Laevens, B. P. M., Martin, N. F., Sesar, B., et al. 2014, ApJL, 786, L3

Law, D. R., \& Majewski, S. R. 2010, ApJ, 714, 229

Luque, E., Queiroz, A., Santiago, B., et al. 2016, MNRAS, 458, 603

Marino, A. F., Milone, A. P., Karakas, A. I., et al. 2015, MNRAS, 450, 815

Martin, N. F., de Jong, J. T. A., \& Rix, H.-W. 2008, ApJ, 684, 1075

Martin, N. F., Geha, M., Ibata, R. A., et al. 2015, MNRAS, 458, L59

Martin, N. F., Ibata, R. A., Collins, M. L. M., et al. 2015, ApJ, 818, 40

Martin, N. F., Nidever, D. L., Besla, G., et al. 2015, ApJL, 804, L5

Milone, A. P., Marino, A. F., Bedin, L. R., et al. 2016, MNRAS, 455, 3009

Milone, A. P., Piotto, G., Bedin, L. R., et al. 2012, A\&A, 540, A16

Muñoz, R. R., Geha, M., Côté, P., et al. 2012, ApJL, 753, L15

Nidever, D. L., Majewski, S. R., Butler Burton, W., \& Nigra, L. 2010, ApJ, 723, 1618

Niederste-Ostholt, M., Belokurov, V., Evans, N. W., et al. 2010, MNRAS, 408, L66

Rosenberg, A., Saviane, I., Piotto, G., Aparicio, A., \& Zaggia, S. R. 1998, AJ, 115,648

Sakari, C. M., Venn, K. A., Irwin, M., et al. 2011, ApJ, 740, 106

Sbordone, L., Bonifacio, P., Marconi, G., Buonanno, R., \& Zaggia, S. 2005, A\&A, 437, 905

Schlafly, E. F., \& Finkbeiner, D. P. 2011, ApJ, 737, 103

Schlegel, D. J., Finkbeiner, D. P., \& Davis, M. 1998, ApJ, 500, 525

Shanks, T., Metcalfe, N., Chehade, B., et al. 2015, MNRAS, 451, 4238

Simon, J. D., Drlica-Wagner, A., Li, T. S., et al. 2015, ApJ, 808, 95

Spitzer, L., Jr., \& Hart, M. H. 1971, ApJ, 164, 399

Taylor, M. B. 2005, adass XIV, 347, 29

The Dark Energy Survey Collaboration 2005, arXiv:astro-ph/0510346

Torrealba, G., Koposov, S. E., Belokurov, V., \& Irwin, M. 2016, MNRAS, submitted (arXiv:1601.07178)

Valdes, F., Gruendl, R. \& DES Project 2014, in ASP Conf. Ser. 485, Astronomical Data Analysis Software and Systems XXIII, ed. N. Manset \& P. Manset (San Francisco, CA: ASP), 379

Villanova, S., Geisler, D., Carraro, G., Moni Bidin, C., \& Muñoz, C. 2013, ApJ, 778, 186

Walker, M. G., Mateo, M., Olszewski, E. W., et al. 2015, ApJ, 808, 108

Walker, M. G., Mateo, M., Olszewski, E. W., et al. 2016, ApJ, 819, 53

Walsh, S. M., Jerjen, H., \& Willman, B. 2007, ApJL, 662, L83

Walsh, S. M., Willman, B., \& Jerjen, H. 2009, AJ, 137, 450

Weisz, D. R., Dolphin, A. E., Skillman, E. D., et al. 2014, ApJ, 789, 147

Weisz, D. R., Koposov, S. E., Dolphin, A. E., et al. 2015, ApJ, submitted (arXiv:1510.08533)

Willman, B., Blanton, M. R., West, A. A., et al. 2005, AJ, 129, 2692

York, D. G., Adelman, J., Anderson, J. E., Jr., et al. 2000, AJ, 120, 1579

Zucker, D. B., Belokurov, V., Evans, N. W., et al. 2006, ApJL, 643, L103 